\title{
Synthesis of Hf-C-N ceramics by spark plasma sintering
}

\author{
Alexey Zavjalov ${ }^{1, *}$, Evgeniy Papynov ${ }^{1,2}$, Oleg Shichalin ${ }^{1,2}$, Pavel Nikiforov ${ }^{1}$, and Evgenii Goncharov ${ }^{1}$ \\ ${ }^{1}$ Far Eastern Federal University, 690090, Vladivostok, Russia \\ ${ }^{2}$ Institute of Chemistry, Far Eastern Branch of Russian Academy of Sciences, 690022, Vladivostok, Russia
}

\begin{abstract}
We obtained the Sample by the spark plasma sintering (SPS) of commercially available HfC and HfN powders. The identification of obtained material was carried out by the X-ray diffraction and the Raman spectroscopy. The solid solution of previous material was confirmed. However oxide phase excreted wile sintering presumably duo to oxygen impurities of used commercially powders.
\end{abstract}

\section{Introduction}

The reasons of the extreme thermal stability of the most refractory compound $\mathrm{Ta}_{4} \mathrm{HfC}_{5}$ (the melting point $\sim 3990 \mathrm{C}$ ) were considered in detail in [1]. The search result for the isostructural analogs of this compound in the Ta-Hf-C-N-B system by the numerical simulation method was the assumption that the melting point of the non-stoichiometric compound $\mathrm{Hf}_{0.53} \mathrm{C}_{0.27} \mathrm{~N}_{0.2}$ is higher up to $200 \mathrm{C}$. Until now, this assumption has not been confirmed experimentally. Therefore, that is the ultimate goal of the authors. The first task on the way to this goal is to obtain Hafnium Carbonitride.

Hafnium Carbide and Nitride have complete mutual solubility and form a solid solutions continuous series [2-6]. The extensive work on obtaining compounds in the Hf-C$\mathrm{N}-\mathrm{O}$ system with different stoichiometry by the vacuum hot pressing method from powders $\mathrm{HfC}, \mathrm{HfN}, \mathrm{HfO} 2, \mathrm{Hf}$, and $\mathrm{C}$ has been carried out for a long ago [2]. The preparation of Hafnium Carbonitride stoichiometric solid solutions mainly occurred during the HfC and $\mathrm{HfN}$ powders sintering while the maximum temperature was $\geq 2300 \mathrm{C}$ for $10-30 \mathrm{~min}$. According to [2], the stoichiometric compound $\mathrm{HfC}_{0.58} \mathrm{~N}_{0.42}$ possesses extreme hardness properties with the ratio $\mathrm{N} / \mathrm{C} \approx 0.72$ close to the ratio of these elements in the proposed in [1] compound $\mathrm{Hf}_{0.53} \mathrm{C}_{0.27} \mathrm{~N}_{0.2}-\mathrm{N} / \mathrm{C} \approx 0.74$. Apparently, such a ratio of the elements in the nonmetallic sublattice best satisfies the revealed in [1] exceptional crystal lattice stability reasons. Therefore, in this work we used the simplest and the closest relation HfN/HfC = $3 / 4=0.75$.

The modern promising method for creating refractory materials is the Spark Plasma Sintering (SPS) (used in this paper). Its advantages over the more classical hot pressing method were demonstrated in [7] for the Ta-Hf-C system. However, the publications about the SPS obtaining materials in the Hf-C-N system are absent. Therefore, the problem of

* Corresponding author: Zav Alexey@list.ru 
obtaining Hafnium Carbonitride by the SPS method from HfC and HfN powders was solved in this study.

\section{Materials and methods}

We used the commercial HfC and HfN powders (Compleks, Russia) ("HfC" and "HfN" respectively in text). The powders mixture (assuming the molar ratio $\mathrm{HfN} / \mathrm{HfC}=3 / 4$ ), was prepared by the planetary mill grinding Pulverisette 6 (Fritsch, Germany) in 6 cycles of 10 min. with a break of $5 \mathrm{~min}$. at $650 \mathrm{rpm}$. The $\mathrm{ZrO}_{2}$ pounder and balls $(6, \varnothing 1 \mathrm{~cm})$ were used while material loading was $\sim 30 \mathrm{~g}$. A homogenization was the goal so the milling parameters were not specially selected. The mixture $(12.12 \mathrm{~g})$ was placed in a graphite crucible and sintered according to the programmed regime in the vacuum (6 Pa) in the SPS515S (Dr. Sinter-LABTM, Japan) to obtain the Sample. The chosen regime based on the related topics [2, 8, 9] and the team experience (for example, [10-13]). The average heating rate was $100 \mathrm{C} / \mathrm{min}$, the maximum sintering temperature was $2100 \mathrm{C}$, the holding time at a maximum temperature of $5 \mathrm{~min}$, and the pressing pressure was constant throughout the process and was $50.4 \mathrm{MPa}$. The test surface of the Sample was first manually cleaned off graphite paper used while sintering by various grain sizes corundum sandpapers (P100P2000 according to ISO-6344) and then ground and polished on the machine MECATECH 234 (PRESI, France) with pre-hot pressing in acrylic resin on the machine MECAPRESS 3 (PRESI, France). The powders and the Sample were investigated on the metallographic microscope Eclipse MA200 (Nikon, Japan). Their phase compositions were identified by the X-ray diffraction carried out on the multipurpose X-ray diffractometer D8 Advance (Bruker, Germany) using $\mathrm{Cu} \mathrm{K}_{\alpha}$ duplet and from the Raman specters obtained on the optical microscope with the Raman spectrometer Morphologi G3SE-ID (Malvern, UK) using Ar-laser wavelength $514.5 \mathrm{~nm}$.

\section{Results and discussions}

The XRD of the Sample and powders are presented on Fig. 1. The double scattering angels investigated range is $5-90^{\circ}$ but Fig. 1 demonstrates only peak-containing sections.

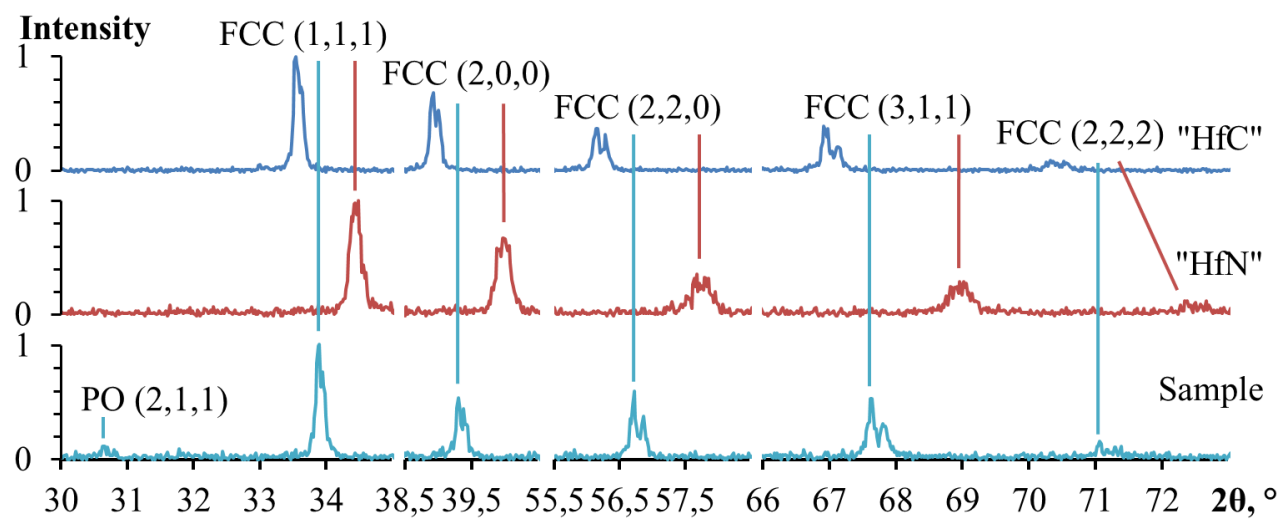

Fig 1. The XRD of powders ("HfC" and "HfN") and the test surface of the Sample.

The Sample peaks lay between the peaks of "HfC" and "HfN" - that proves the solid solution obtaining. The XRD indexing uniquely identifies the face-centered cubic (FCC) lattice. The $\mathrm{Cu} \mathrm{K}$ duplet reflexes are clearly distinguishable. The precise positioning of the peaks further the second is difficult so the lattice constants were calculated only from first 


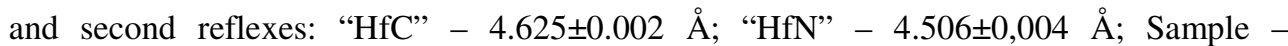
$4.582 \pm 0.003 \AA$. The lattice constants of "HfC" and "HfN" are less than the standards: HfC $-4,638 \AA$ [14]; HfN - 4,525 $\AA$ [15]. This is typical for defective structures - for example, with some oxygen impurities. If the linear dependence between a lattice constant and electronegativity is assumed then the lattice constant of fictive phase 'HfO' will be estimated $(\sim 4,416 \AA)$ as well as (adopting Vegard's rule) the share of the substituting oxygen: $\sim 6 \%$ for "HfC" and $\sim 18 \%$ for "HfN".

According to optical microscopy (Fig. 2.a), the grain size and coarse pore fraction correspond to the dispersion of the powders (not presented) $-10-20 \mu$. The fine pores fraction $(\sim 1-3 \mu)$, apparently, is the intrinsic porosity of the sintered particles, which is typical for self-propagating high-temperature synthesis of powders. Two phases are clearly visible on the photomicrograph (Fig. 2.a). The light main phase corresponds to FCC peaks (Fig. 1). The dark phase can be correlated only with a weak peak close to the reflex (211) Primitive orthorhombic (PO) $\mathrm{HfO}_{2}$ [17]. The dark phase identification was carried out by Raman spectroscopy (Fig. 2.b).

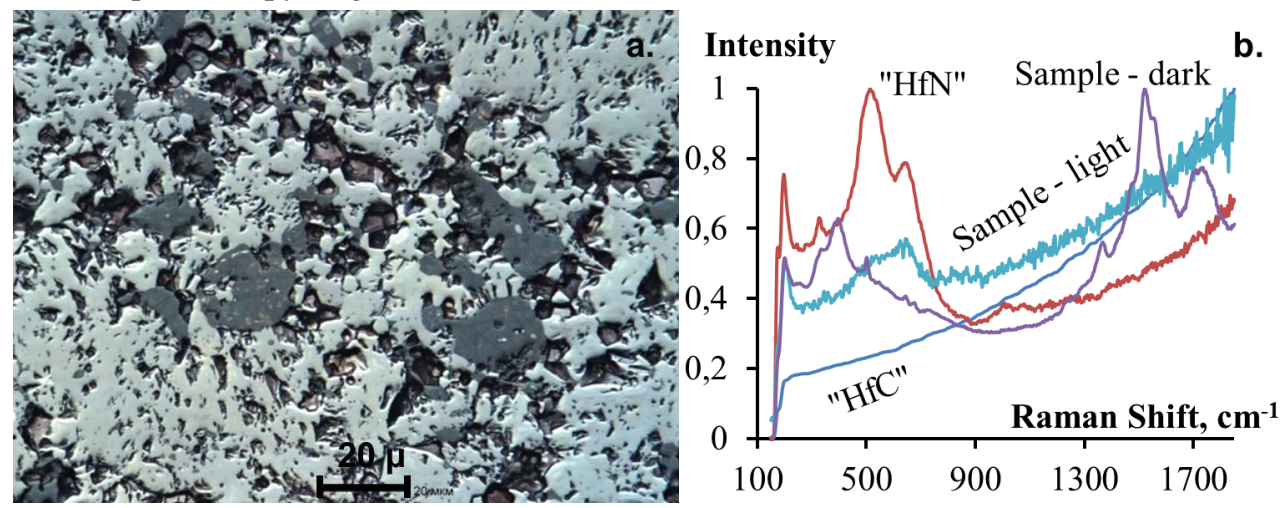

Fig 2. a. The Sample test surface microphoto. b. The Raman spectra for powders and Sample phases.

The porosity preservation indicates the sintering without significant softening which is fully corresponds to a low sintering temperature $(\sim 2100 \mathrm{C})$ in comparison with the melting points of HfC $(\sim 3890 \mathrm{C})$ and $\mathrm{HfN}(\sim 3380 \mathrm{C})$. The formation of a new solid solution phase occurred during the mutual diffusion of $\mathrm{C}$ and $\mathrm{N}$. It required lower temperatures in comparison with the SPS production of $\mathrm{Ta}_{4} \mathrm{HfC}_{5}[8,9](\geq 2350 \mathrm{C})$, which may be explained by the facilitated diffusion of $\mathrm{C}$ and $\mathrm{N}$ due to their smaller masses and sizes (v.s. Ta and $\mathrm{Hf}$ ).

"HfC" and the main (light) phase do not have a pronounced spectrum in the investigated range of Raman shifts. The "HfN" lines correspond to HfN with a defective structure [18], which was also revealed by Fig.1. The implicitly expressed broad peak of the light phase $\left(\sim 400-750 \mathrm{~cm}^{-1}\right)$ can be associated with the HfN lines. The Raman spectrum of the dark phase corresponds to $\mathrm{HfO}_{2}$ [19]. Deviations are explainable by the presence of defects.

\section{Conclusions}

This study task was to obtain Hafnium Carbonitride by the SPS. The oxygen admixture in the used powders led to the additional oxide phase separation. However, there is no reason to believe that oxygen plays a key role in sintering. Thus, the posed task can be considered as solved. The material was obtained at lower temperatures $(\sim 2100 \mathrm{C})$ and holding time $(5$ min) than used in more traditional methods [2-4] ( $\geq 2300 \mathrm{C}, \geq 10 \mathrm{~min})$. Further, the SPS method will allow to obtain the qualitative Hafnium Carbonitride ceramic materials by 
more carefully selecting the sintered powders and preparing samples for testing the hypothesis about more refractory compounds in the $\mathrm{Hf}-\mathrm{C}-\mathrm{N}$ system than $\mathrm{Ta}_{4} \mathrm{HfC}_{5}$.

The study was carried out at the Russian Science Foundation grant expense (project № 18-73-00125).

\section{References}

1. Q. Hong, Phys. Rev. B 92, 020104(R) (2015)

2. G.D. Brundiers, Herstellung, Aufbau und Eigenschaften von Hafniumverbindungen im System Hf-C-N-O (Gesellschaft für Kernforschung M.B.H., Karlsruhe, 1975)

3. S. Binder et al., J. Alloys Compd. 217, 128 (1995)

4. W. Lengauer et al., J. Alloys Compd. 217, 137 (1995)

5. J. M. Córdoba et al., J. Am. Ceram. Soc. 90, 381 (2007)

6. W. F. Piedrahita et al., J. Alloys Compd. 690, 485 (2017)

7. V.V. Kurbatkina et al., Ceram. Int. 44, 4320 (2018)

8. O. Cedillos-Barrazaa et al., J. Eur. Ceram. Soc. 36, 1539 (2016)

9. S.A. Ghaffari et al., J. Eur. Ceram. Soc. 33, 1479 (2013)

10. E.P. Simonenko et al., J. Sol-Gel Sci. Technol. 82, 748 (2017)

11. E.P. Simonenko et al., Russ. J. Inorg. Chem. 61, 1203 (2016)

12. V.G. Sevastyanov et al., Russ. J. Inorg. Chem. 60, 1360 (2015)

13. V.G. Sevastyanov et al., Russ. J. Inorg. Chem. 59, 1298 (2014)

14. W. Wong-Ng et al., Powder Diffr. 3, 113 (1988)

15. M.C. Morris et al., Pyrros standard X-ray Diffraction Powder Patterns. Section $19-$ Data for 51 Substances (U.S. Government Printing Office, Washington, 1982)

16. G.G. Filippov, A.I. Gorbunov, Russ. J. Gen. Chem. 39, 39 (1995)

17. The ICDD Database, Data set number: 01-083-0808

18. M. Stoehr et al., J. Appl. Phys. 104, 033507 (2008)

19. C.W. Li, M.M. McKerns, B. Fultz, Phys. Rev. B 80, 054304 (2009) 